Proceedings of the 2009 Winter Simulation Conference

M. D. Rossetti, R. R. Hill, B. Johansson, A. Dunkin, and R. G. Ingalls, eds.

\title{
A WEB SERVICES BASED ARTIFICIAL MARKET
}

\author{
Karla Atkins \\ Chris Barrett \\ Achla Marathe \\ Virginia Bioinformatics Institute \\ Virginia State University and Polytechnic Institute \\ Blacksburg, VA, 24061 USA
}

\begin{abstract}
We describe a web services based computational tool for studying large commodity markets. The software architecture is based on three important guiding principles: (i) efficiency and scalability, (ii) extensibility to incorporate several different clearing mechanisms and commodities, (iii) modularity for platform independent operations and use. The computational model has several distinguishing features. They include: (i) the ability to generate individualistic, demographics based, time-varying demand profiles, (ii) a highly configurable system that supports different market clearing mechanisms, strategies and matching algorithms for buyers and sellers (Atkins, Marathe, and Barrett 2007), (iii) ability to aggregate individuals into different hierarchy of classes and (iv) ability to physically clear flow based commodities. From the software standpoint, the architecture has several unique features, including use of web services for loosely coupling individual elements of the system, an easy to use web based graphical user interface for specifying input parameters as well as viewing the results, and a work flow language for modeling various markets and market mechanism (Atkins et al. 2004). The system provides users the unique ability to experiment with a variety of markets such as market for communication spectrum, Internet bandwidth, electricity, as well as traditional commodities like corn, cotton etc.
\end{abstract}

\section{THE MARKET MODELING FRAMEWORK}

We describe a web services based computational tool for studying commodity markets. SIGMA (Simulation of Generic Markets) is a service oriented, high fidelity, agent-based, computational market modeling tool. The software architecture is based on three important guiding principles: (i) efficiency and scalability, (ii) extensibility to incorporate several different clearing mechanisms and commodities, (iii) modularity for platform independent operations and use. More specifically, SIGMA functionality is facilitated and enhanced through the following software based features.

- Service Oriented Architecture. SIGMA is based on a service oriented architecture. The choice of this architecture was natural since we wanted the system to be usable by a larger community via the web. The architecture is globally scalable: multiple users can use the system simultaneously. We believe that it provides the first step to integrate the software tool with a grid computing paradigm in the near future. The second aspect of scalability has to do with the size of models that our system can handle. Our analysis shows the system can easily handle over a million players. The scaling is achieved through implementation of efficient algorithmic and software engineering constructs.

- Flexible and Extensible Design. The economic literature consists of numerous market mechanisms, auctions, trading strategies, and clearing rules. From the software perspective, the goal was to develop a generic software framework which allows the users to play with different market components as well as add new features to the software. We illustrate this by incorporating configurable classes of buyers and sellers, trading institutions and physical clearing options. The extensibility is necessary since many markets are evolving and scientist from time to time propose new market mechanisms for constructing efficient markets. For example, the researchers have proposed numerous market mechanisms for trading contracts in the deregulated electricity market and spectrum markets (Barrett et al. 2003). 


\section{Atkins, Barrett and Marathe}

- Flexible Work-flow Language. SIGMA has a flexible work-flow language that serves as a way to generate different models as specified by the user. Note that SIGMA is a modeling environment rather than an implementation of a specific model. Different models can be created composing i) specific models for buyers and sellers ii) specific ways in which markets are cleared, and iii) specific ways in which market operations are scheduled and combined.

- Web Based GUI. In order to allow non-specialist computer users easy access to SIGMA's modeling capabilities, a web based graphical user interface has been designed. The graphical user interface allows the user to specify model parameters and work-flow by generating configuration files. It offers the user the opportunity to combine individual configurations into scenarios such that entire studies can be composed. SIGMA, which dynamically creates the models embedded in each configuration, may be executed next. Finally, results may be plotted, or data files that can be easily analyzed may be downloaded to the user's computer.

SIGMA has several unique features. The most salient are:

- Individual based Demand Modeling. SIGMA allows development of individualistic demand models. Given that different individuals have different preferences, objectives, desires and priorities, it is important to capture the individualistic behavior to keep the model realistic. The advent of computer-based modeling allows us to model the heterogeneity of the individuals and drop many of the traditional constraints while maintaining the feasibility of the calculations. In contrast, under most current modeling techniques, characteristics of the population are aggregated and the model attempts to simulate changes in the averaged characteristics for the entire population. The demand profiles in SIGMA are mobility and activity based, price and time dependent, and include information about the demographics of the population (Atkins, Marathe, and Barrett 2007). Examples of commodities wherein such modeling is useful include electricity and spectrum trading. Agents in SIGMA also do not have to be perfectly rational and driven by pure self interest.

- Coupling with Physical Networks and Clearing Mechanism. SIGMA has the flexibility to be coupled with various physical clearing mechanisms. The flow based commodities require physical infrastructure for trading. For these commodities mere economic contracting between the buyers and sellers does not ensure the feasibility of physical clearing. Examples of commodities that require physical clearing are gas, water, and electricity. For these flow based commodities physical flow models are required to ensure economic contracts can actually be delivered. SIGMA is currently coupled with a power flow model to clear the electricity contracts. Other flow models can be added as necessary. Classical commodities such as wheat, cotton and corn that are transported over a network can also be modeled.

- Auction Mechanisms. SIGMA is designed so that the markets can be cleared through various auction mechanisms. It currently offers English (first price) auction, and Vickrey (second price) auction. The configurable market framework allows users to study various auction designs, their performance in terms of computability, practicality, and their ability to efficiently allocate resources.

- Input from Human and Computer Agents. The service based architecture is also designed to allow participation by the users wherein the users can participate in the market by sending bids and asks and interacting with it in real time. We envisage a system that is flexible enough to use input either solely from the computer agents as is commonly done in the agent based systems or solely from the human agents as is done in human-based laboratories and classrooms or a combination of both computer agents and human agents.

- Educational Tool. Its web based design makes it particularly easy to be used as an educational tool by researchers and students. In this virtual laboratory one can explore and understand the complex operations of a market and agents' behavior.

\section{RELATED WORK}

There has been substantial work in the area of developing agent based computational models for various socio-economic problems. The experimental work in markets can be partitioned into two broad classes. One is the human-based laboratory experiments, in which experiments are performed in a controlled environment and the cash-motivated human subjects are used as agents (Olson et al. 2001, Gode and Sunder 1993, Plot and Sunder 1988, Smith 1962). Human-based laboratories provide a natural environment for individual preferences and their reaction to institutional and regulatory changes to be studied. However, there are several limitations on the kinds of experiments that can be performed using human subjects. For example, inability to scale to a realistic number of players, set up complicated 'rules of the game', play out collusive and learning behavior etc. 

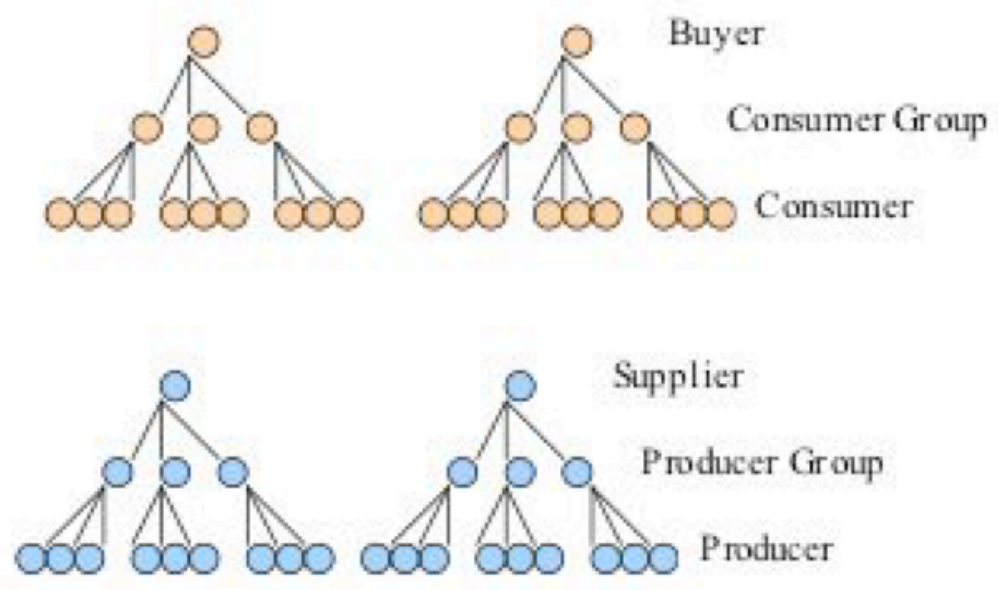

Figure 1: Market Player Hierarchies

The other class involves the computer-based experiments which use agent based computational models (Rust et al. 1994). These models can address a number of complex and interrelated issues that cannot be resolved using conventional economic models which are capable of providing solutions only in analytically tractable ways. Computational models can also be scaled to more realistic levels. California's experience with deregulation of the electricity industry demonstrated some of the risks that markets can get exposed to when the design issues are not well thought of. A simulation based computational model will be very helpful in providing a deeper understanding of the issues related to new market designs as well as in analyzing markets that are still in transient.

Human behavior depends on a multitude of social, economic, demographic, cultural and psychological factors. Work in social, behavioral and economic sciences has focused on developing detailed models of human behaviors under varying circumstances. This includes work in which humans are modeled as rational agents and recent research that challenges this hypothesis and develops models that are behavioral and thus not necessarily rational. This includes classical results by (Camerer 1999, Tversky and Kahneman 1973). The agents' behavior displays human limitations, complications and possibly deviation from the classical rationality assumptions. A large and growing body of scientific work in economics and behavioral sciences is now devoted to empirical testing and modifications of traditional assumptions such as unbounded rationality, pure self interest, exponential discounting etc. Some of the work in behavioral sciences that has challenged the fundamental assumptions in classical economics can be found in (Rubenstein 2001, Smith 1991, Tversky and Kahneman 1973, Roth 1993). Although a lot of work has gone in developing these models, very little work has gone in the development of computational models and software systems that study the effect of interaction between individual agents that show such behavior. The web service based architecture of SIGMA hopes to fill this gap by allowing participation from both the computer and human agents.

\section{ARCHITECTURE OVERVIEW}

SIGMA consists of following main conceptual components: (i) consumers (buyers) (ii) producers (sellers), (iii) market representation and market specific rules. The users can control player preferences, player strategies, model assumptions, initial market conditions and trading mechanisms. A detailed representation of consumers, producers and markets is used. From a microscopic, disaggregated structure, individual consumers and producers are aggregated into hierarchies of buyers and suppliers respectively. Flexible capabilities for aggregation of demand and supply profiles allow instant creation of various market types, including retail, wholesale, competitive, oligopoly, and monopoly markets. The current framework also offers centralized and decentralized economic clearing options along with a real-time (spot) market. The real-time market is required for commodities such as electricity. 


\section{Atkins, Barrett and Marathe}

The above conceptual structure is represented within the software architecture using the following software components:

- Consumer: A consumer has the following defining attributes: (i) demographic profile, (ii) specific functions for generating time varying, location and income dependent demand as a function of price, (iii) targeted demand. A set of consumers can be aggregated to form a consumer group.

- Buyer: The consumer groups can be further aggregated to make a buyer. The buyers are the economic representative of the consumer groups and consumers in the market and make bids on their behalf.

- Producer: A producer has the following defining attributes: (i) estimated demand in the market, (ii) production cost function, (iii) bidding strategy (iv) geographic attribute (if relevant). Just like consumers, a producer group can be formed by aggregating a number of producers.

- Supplier: The producer groups can be further aggregated to make a supplier. The suppliers are the economic representative of the producers and producer groups in the market and send asks on their behalf.

- Market. Three kinds of markets are explicitly modeled; centralized, decentralized and real-time market. The framework allows configuration of many different kinds of markets by the user. For example, different levels of consumer and producer aggregation can create competitive and monopolistic markets.

- Work-flow Module. The simulation is executed through a work-flow module which gives user the ability to configure a market with specific parameters, level of consumer and producer aggregation, style of market clearing etc.

- GUI Interface. Users with little or no knowledge of markets and computers can use SIGMA's modeling capabilities through the web based graphical user interface. The graphical user interface allows the user to specify model parameters and work-flow through configuration files.

- Physical Clearing Module. Different physical flow models can be coupled with the market model to ensure physical clearing of the economic contracts. Currently SIGMA is coupled with a power flow model that computes the load of each transmission line on the grid. The economic contracts that result in load of a line to exceed the capacity of the line get rejected since they are not physically feasible.

\subsection{Market Players and their Inter-Relationships}

Market players are individual producers and consumers with their own supply and demand functions. In commodity markets an individual player may be served indirectly through a buyer or a supplier agent. Each buyer and supplier may represent multiple individuals. The use of flexible mechanisms for aggregation of market players into hierarchies with corresponding methods for aggregation of supply and demand functions allows SIGMA to readily model a variety of market types, while keeping requirements for computational resources manageable. For example, a monopoly can be created through aggregation of all producers under a single supplier. The figure 1 illustrates market player hierarchies in SIGMA.

\subsection{SIGMA Schedules and Resulting Work Flow}

The flow of work in a SIGMA execution is straightforward. A simulation is driven by a flexible user-specified schedule that designates (among other things)

- The number of simulated hours desired

- The forward and real-time markets to run within an hour

- The number of iterations for each market within an hour

- The physical clearing model to use, if any

- The frequency of physical clearing, if appropriate

Within a market execution, the paradigm is

- Do economic clearing

- Do physical clearing of the economically cleared markets if requested in the schedule

- Do bookkeeping

The SIGMA schedule is input in an XML file, which may be optionally created by a web-based GUI. Once parsed by SIGMA, it is stored in a data structure comprised of a collection of related objects as shown in the following diagram. SIGMA produces output on a per-iteration, an hourly, and a per-run basis. The per-iteration data output is the result of the "Do bookkeeping" step of a given market execution. The hourly data output is created by the main schedule run method. The 


\section{Atkins, Barrett and Marathe}

per-run data output is the result of the SIGMA main program after the schedule has been run. SIGMA input demand data is organized by the hour. Accordingly, SIGMA implements hourly reset or initialization operations through which hourly input data are loaded. Like hourly output operations, the reset process is initiated by the main schedule run method.

\subsection{Supplier Bidding Strategies}

A SIGMA supplier agent can be configured with a competitive, oligopolistic, or an intermediate strategy. These strategies are based on economic profit maximizing theory (Atkins, Marathe, and Barrett 2007). Additional strategies will be added that will allow studies in the behavioral economics field. The profit maximizing method used by SIGMA requires that supplier agents carry their own estimation of how consumers will behave in the market. This is accomplished using an estimated demand function. The estimated demand function is adjusted each hour using a factor based on the expected market share relative to the realized market share.

\subsection{Supplier Learning Behavior}

The suppliers have been endowed with some learning abilities. Based on their past performance in terms of profits and market share, the suppliers can adjust their bidding strategies in the future bids. The goal is to address some of the issues raised in the "Profits vs. Market Share" debate. There are two basic models followed in the financial world. First, maximize profits as the classic American model suggests. Second, capture the market share by under-cutting the competitors' prices. The second model was pioneered by the Japanese in the 1960s who were more market share conscious. Japanese corporations often tolerate lower profits for the sake of getting higher market share (George 1999). However, the American enterprises are focused on maximizing profits because the share holders are mainly driven by big profit margins.

In the SIGMA framework, the suppliers can bid in the market based on one of the three strategies i.e. a competitive strategy, oligopolistic strategy, or an in between strategy called competitive-oligopolistic. Under the competitive-oligopolistic strategy, the bidding price is chosen by uniformly randomly selecting a price between the profit maximizing competitive price and the profit maximizing oligopolistic price. Similarly, the suppliers can select from one of the two objectives i.e. maximize profits or maximize market share. The supplier may be willing to sacrifice profits in order to capture market share and broaden his revenue base. Once the supplier has captured a desired level of market share, a more aggressive strategy can be followed to attain a higher clearing price in the market. As more market share is captured, the supplier's influence on the market increases, allowing him to be more aggressive in the bidding process.

\section{MARKET MECHANISMS}

This section summarizes the explicitly modeled market clearing mechanisms.

\subsection{Decentralized Market}

The "BilateralMarket" module of SIGMA models the decentralized market. In a decentralized market, buyers and sellers independently negotiate the financial and physical terms of a contract. As such, multiple clearing prices and quantities are in effect. In SIGMA, decentralized contracts are issued using the intersection of a supplier's total average cost curve and a buyer's demand curve. Potential contracts that do not meet boundary conditions imposed by the buyers' target demand, market price cap, and supplier ask quantity are rejected.

SIGMA offers a variety of methods for matching buyers to suppliers prior to determination of contract feasibility and subsequent contract generation. Specific options include:

- Randomly match buyers and suppliers based on a user-specified probability.

- Selectively match buyers and suppliers, depending on user-specified criteria pertaining to agent attributes, such as quantity asked, price asked, and target demand.

- Arbitrarily match buyers and suppliers using user-specified order pertaining to buyer and supplier index (id) values. Nine orders are supported: (i) Buyer ascending, Supplier ascending (ii) Buyer random, Supplier ascending, (iii) Buyer descending, Supplier ascending, (iv) Buyer ascending, Supplier random, (v) Buyer random, Supplier random, (vi) Buyer descending, Supplier random, (vii) Buyer ascending, Supplier descending, (viii) Buyer random, Supplier descending, and (ix) Buyer descending, Supplier descending. 


\section{Simulation of Generic Markets (SIGMA)}

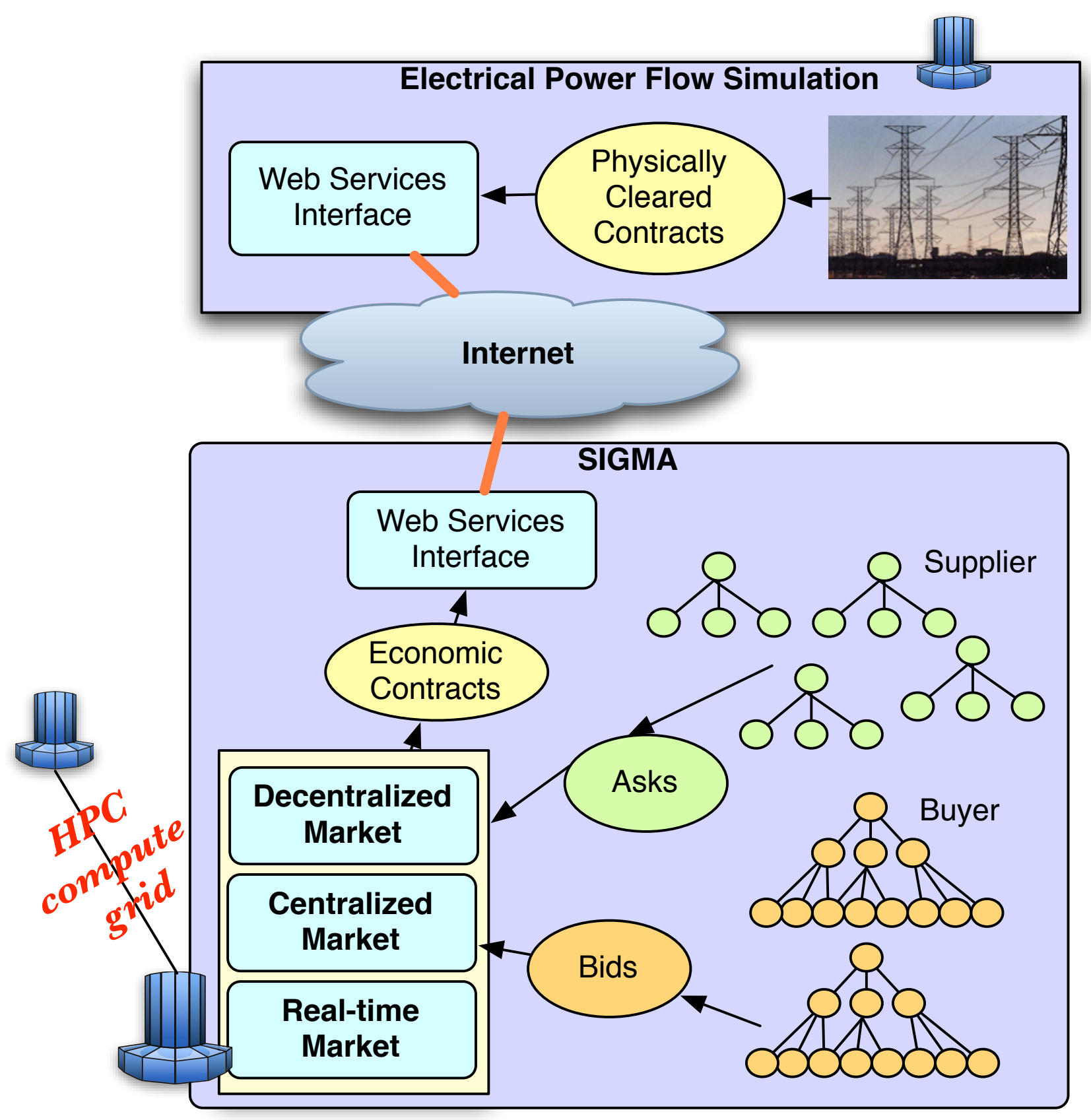




\section{Atkins, Barrett and Marathe}

A case study has shown that the order of matching is highly significant in a simulated decentralized environment (Atkins, Marathe, and Barrett 2007).

\subsection{The Centralized Market}

The "PoolcoMarket" module of SIGMA models the centralized market. In a centralized market all trades occur at a uniform market clearing price. In a centralized market, buyers and sellers submit bids and asks to an exchange, and the exchange dispatches asks in economic order (lowest price ask is dispatched first) until all bids are satisfied or supply is exhausted. The SIGMA implementation uses a horizontal summation of consumer demand curves intersecting with a set of strategy dependent, profit maximizing bids and asks (subject to constraint conditions, e.g. market price cap), to determine a market-wide clearing price and quantity.

\subsection{Real-time Market}

The Real-time market models a real-time or spot market. In a real-time market, the unsatisfied demand and supply of the forward markets is cleared. The markets described above i.e. the centralized and the decentralized markets are the forward markets in SIGMA.

\subsection{Case Studies}

Applicability of this framework has been demonstrated through a variety of case studies. For example (Atkins, Marathe, and Barrett 2007) study shows the order in which the buyers and sellers are matched in a bilateral market makes a big difference in trading price and volume among other things. Work in (Atkins et al. 2009) and (Atkins, Marathe, and Barrett 2007) shows that strategic alliances among small generators with geographical advantage can lead to significant amounts of locational market power. However, the market power can be curtailed by making demand more responsive to price.

\section{SUMMARY}

In this paper, we present a service based tool and modeling framework for analyzing large scale commodity markets. We demonstrate the applicability of our computational framework, its generic features, flexible architecture and hierarchical representation. It provides a controlled laboratory setting where the user can test key economic assumptions, theories and policies in a controlled environment. The service oriented design enables the user to specify the model parameters in detail and study the markets as they move from a transient state to a steady state. Such transients cannot be studied through traditional closed form economic models.

\section{ACKNOWLEDGEMENTS}

This work has been partially supported by NSF Nets Grant CNS- 062694, NSF HSD Grant SES-0729441 and DTRA CNIMS Grant HDTRA1-07-C-0113.

\section{REFERENCES}

Atkins, K., C. Barrett, C. Homan, A. Marathe, M. Marathe, and S. Thite. 2004. Agent based economic analysis of deregulated electricity markets. In 6th IAEE European Conference. Zurich, Switzerland: IAEE.

Atkins, K., J. Chen, A. Kumar, M. Macauley, and A. Marathe. 2009, May. Locational market power in network constrained markets. Journal of Economic Behavior and Organization 70 (1-2): 416-430.

Atkins, K., A. Marathe, and C. Barrett. 2007, September. A computational approach to modeling commodity markets. Computational Economics 30 (2): 125-142.

Barrett, C., D. Cook, V. Faber, G. Hicks, A. Marathe, M. Marathe, A. Srinivasan, Y. J. Sussmann, and T. Heidi. 2003. Experimental analysis of algorithms for bilateral-contract clearing mechanisms arising in deregulated power industry. Journal of Graph Algorithms and Applications 7 (1): 3-31.

Camerer, C. 1999, September. Behavioral economics: Reunifying psychology and economics. Proceedings of National Academy of Sciences 96.

George, W. 1999. Apple's future: Profits vs. market share. http://www.macobserver.com/columns/appletrader/99/march/990322.html. 


\section{Atkins, Barrett and Marathe}

Gode, D. K., and S. Sunder. 1993. Allocative efficiency of markets with zero-intelligence traders: Market as a partial substitute for individual rationality. Journal of Political Economy 101 (1): 119-137.

Olson, M. A., S. J. Rassenti, V. L. Smith, M. L. Rigdon, and M. J. Ziegler. 2001. Market design and motivated human trading behavior in electricity markets. IIE Transactions on Operations Engineering.

Plot, C. R., and S. Sunder. 1988. Rational expectations and the aggregation of diverse information in laboratory security markets. Econometrica 56 (5): 1085-1118.

Roth, A. L. 1993, October. Rationality in economics'. In IEA Conference. Torino, Italy.

Rubenstein, M. 2001, May-June. Rational markets: Yes or no? the affirmative case. Financial Analysts Journal.

Rust, J., J. H. Miller, and R. Palmer. 1994. Characterizing effective trading strategies: Insights from a computerized double auction tournament. Journal of Economic Dynamics and Control 18:61-96.

Smith, V. L. 1962. An experimental study of competitive market behavior. The Journal of Political Economy 70 (2): 111-137.

Smith, V. L. 1991. Rational choice - the contrast between economics and psychology. The Journal of Political Economy 99:877897.

Tversky, A., and D. Kahneman. 1973. Availability: A heursistic for judging frequency and probability. Cognitive Psychology 5:207-232.

\section{AUTHOR BIOGRAPHIES}

KARLA ATKINS received her B.S. in Information Systems in 1980. She worked as a Senior Research Associate at the Network Dynamics and Simulation Science Laboratory within Virginia Tech's Virginia Bioinformatics Institute. Her current research interests include advanced software architectures, modeling and simulation of complex economic and other socio-technical systems, grid-based and parallel computing and related data management strategies. Her email address is $<$ katkins10@comcast.net>.

CHRIS BARRETT is a professor of Computer Science, and the Director of Network Dynamics and Simulation Science Laboratory at Virginia Tech. He received his Ph.D. in 1985 in Bioinformation Systems from the California Institute of Technology. His research interests include interaction based systems, computing and dynamical systems, infrastructure simulation, and computational epidemiology. His email address is <cbarrett@vbi.vt.edu>.

ACHLA MARATHE is the lead economist at the Network Dynamics and Simulation Science Laboratory at Virginia Tech. She is also an associate professor at the Agriculture and Applied Economics department at Virginia Tech. Her research interests include modeling and simulation of socio-economic systems, computational economics and restructured electricity markets. Her email address is <amarathe@vbi.vt.edu>. 